\title{
Some Aspects of Complex Quantum Theories: A Brief Note
}

\author{
Bhabani Prasad Mandal \\ Department of Physics, Institute of Science, Banaras Hindu University, Varanasi -221005, India \\ Email: bhabani.mandal@gmail.com
}

\begin{abstract}
Theories with indefinite metric have a long and very rich history. Such theories are being used time to time in searching the solutions for many outstanding problems in physics over the several decades. However much attentions were not paid for these as they were not mathematically consistent with usual quantum theories. Towards the end of last century this field of research received huge boost, when attempts had been made to accommodate certain class of non-Hermitian theories in rigorous mathematical formulation. Subsequently it has been shown that certain specific types of non-Hermitian theories can lead to fully consistent quantum theories with complete real spectrum, unitary time evolution and probabilistic interpretation in a modified Hilbert space. Due to this important realisation, theories with complex Hamiltonian have become the topic of frontier research over last two decades and enormous applicationswere found in various branches of physics. In this brief note we try to review certain aspects of such theories where we have contributed. In particular, we discuss PT phase transition in non-relativistic, relativistic and quantum field theoretic systems and some new features of scattering in complex systems.
\end{abstract}

Keywords: Quantum theories, Non-Hermitian formulations, PT phase transition.

\section{Introduction}

In 1998, Bender et al [1] showed that a class of non-Hermitian system can produced complete real spectrum. This motivates to develop a consistent quantum theory with unitary time evolution and correct probabilistic interpretations for certain class of non-Hermitian systems. Subsequently fully consistent quantum theory by restoring the equivalent Hermiticity and upholding the unitary time evolution for parity $(\mathrm{P})$ time reversal (T) invariant non-Hermitian systems is developed in a modified Hilbert space endowed with CPT inner product. The operator $\mathrm{C}$ is analogous to charge conjugation symmetry and connects the states with PT negative and positive norm states. Later this formulation was put in firm mathematical basis for a bigger class of non-Hermitian system, known as pseudo-Hermitian system [2]. The details of these formulations are written in two excellent review articles [3,4]. Many of the predictions of such theories have been verified experimentally in analogous classical systems [5]. These experimental realisations of non-Hermitian systems in laboratories prompted huge interests in the study of non-Hermitian systems both in theory and in experiments and rapidly developed over variousbranches of physics [6]. We are not attempting to account all 
important features of such theories in his brief note, rather present two of the major characteristics of these theories. NonHermitian PT symmetric systems generally exhibit a phase transition that separates two parametric regions (i) region of the unbroken PT symmetry in which the entire spectrum is real and eigenstates of the systems respect PT symmetry and (ii) a region of the broken PT symmetry in which the whole spectrum (or a part of it) appears as complex conjugate pairs and the eigenstates of the systems do not respect PT symmetry. The study of PT phase transition has become extremely important due to the fact that such phase transition and its rich consequences are really observed in variety of physical systems [5]. On the other hand the scattering due to complex potential has many rich features like exceptional points, spectral singularity (SS), invisibility, reciprocity, coherent perfect absorption (CPA), critical coupling (CC) etc. The investigations in these aspects have enriched the study of different optical systems. In this brief note we will highlight our results mainly in these two directions.

\section{PT Phase Transition}

We worked PT phase transition in various models in relativistic and non relativistic quantum theories as well as in models using quantum field theories. We plan to outline the essential features of these works in this section. In non-relativistic quantum mechanics we for first time have considered a model in more than one dimensions [12]. In $2+1$ dimension we consider an anisotropic oscillator with imaginary $i x y$ interaction and is described by,
$H=\frac{p_{x}^{2}}{2 m}+\frac{p_{y}^{2}}{2 m}+\frac{1}{2} \omega_{x}^{2} x^{2}+\frac{1}{2} \omega_{y}^{2} y^{2}+i \lambda x y$

where $\lambda$ is real. This model is exactly solved by analytical method to observed the following. (i) When the coupling, $\lambda \leq \lambda_{\mathrm{c}}$, a critical value of coupling, the entire spectrum is real and eigen functions of the Hamiltonian respect to PT symmetry i.e. the system is in unbroken phase. (ii) for $\lambda>\lambda_{c}$ the whole spectrum or a part of it becomes complex and the system is in broken phase. (iii) The critical coupling $\lambda_{c}$ depends on the anisotropy of the system and the phase transition ceases to exist when the system becomes isotropic. The interesting observation is that the rotational symmetry of the original Hermitian system is complementary to the unbroken PT symmetry in the non-Hermitian model. In an another model we considered an isotropic SHO with charge $\mathrm{q}$ in $3 \mathrm{~d}$ in presence of an external imaginary magnetic field along $\mathrm{z}$ direction described by the Hamiltonian,

$H=\frac{1}{2 m}\left(\vec{p}-\frac{i q \vec{A}}{c}\right)^{2}+\frac{1}{2} m \omega^{2}\left(x^{2}+y^{2}+z^{2}\right)+$ $i \vec{\mu} \cdot \vec{B}$

The use of imaginary magnetic field is motivated by a study on localization and delocalization phase transition in superconductor [20]. We showed that the system undergoes a PT phase transition when the strength of the magnetic field $B \geq B_{c}$, the critical magnetic field. Alternatively for a fixed magnetic field, PT phase transition occurs when the frequency $\omega$ of harmonic oscillator becomes half of the cyclotron frequency $\omega_{c}$. At the transition point the oscillator do not have any dynamics in $x-y$ plane, it only oscillates in the direction of 
magnetic field with its original frequency. We would further like to add that the imaginary magnetic field along a preferred direction ( $\mathrm{z}$ direction) creates the anisotropy in this three dimensional model. In the relativistic model [13] we have studied a massless Dirac particle with PT symmetric non-Hermitian Rashba interaction in the background of Dirac oscillator potential to show the PT phase transition in $(2+1)$ dimension analytically. The model is described by the Hamiltonian,

$$
\begin{aligned}
& H=v_{f}\left[\vec{\sigma} \cdot\left(\vec{p}+\frac{e \vec{A}}{c}-i K_{1} \vec{r} \beta\right)+\right. \\
& \left.i \lambda\left(\vec{\sigma} \times\left(\vec{p}+\frac{e \vec{A}}{c}\right)\right) \cdot \hat{z}\right]
\end{aligned}
$$

$\mathrm{K}_{1}, \lambda$ are real constant, $\mathrm{v}_{\mathrm{f}}$ is Fermi velocity. We have shown with analytical calculation, explicitly that PT phase transition occurs when strength of the (i) imaginary Rashba interaction or (ii) transversemagnetic field exceed their respective critical values. Small mass gap in the spectrum of massless Dirac particle, consistent with other approaches is generated as long as the system is in the unbroken phase. Relativistic Landau levels are constructed explicitly for such a system. It will be exciting to correlate some of the properties of graphene with the PT phase transition. In a recent work [14], we have realised deconfinement to confinement phase transition in $\mathrm{SU}(\mathrm{N})$ QCD as a PT phase transition. For this purpose, we considered the model described by the Lagrangian density,

$L=\frac{-1}{4} F_{\mu v}^{a} F^{a \mu v}+\frac{\xi}{2} F^{a 2}+F^{a} A_{\mu}^{a} A_{v}^{a}-$
$2 \overline{c^{a}} A^{\mu a}\left(D_{\mu} c\right)^{a}$
By considering natural hermiticity property of the ghost fields we cast this model as nonHermtian but symmetric under combined Parity (P) and Time reversal (T) transformations. We explicitly study the PT phase transition in this model. This is very first such study in the non-Hermitian gauge theory. The ghost fields condensate as a direct consequence of spontaneous breaking of PT symmetry. This leads to realize the transition from deconfined phase to confined phase as a PT phase transition in this system. The hidden C- symmetry, which is inherent in all PT symmetric non-hermitian model is identified as inner automorphism in this theory. Explicit representation is constructed for the $\mathrm{C}$ symmetry.

\section{Complex Scattering}

Scattering due to complex potential has many exciting properties. Among these CPA which is the time reversal of lasing effect is central to all studies in complex scattering and can be observed in scattering when wave interact with surrounding medium through a complex potential distribution. Further the investigation on CPA is largely boosted due to the discovery of anti-Laser in which incoming beams of light interfere with one another in such a way as to perfectly absorb by the medium [21]. One major problem in constructing Anti-Laser in laboratory is that CPA happens only at a specific value of the energy of the incident beam. We have made an attempt to overcome this limitation by complexifying 1D Hulthen potential [17] and Wood-Saxsen (WS) potential [18]. Byadjusting the parameters we have achieved CPA almost for the entire range of incident 
energy in the case of Hulthen potential and obtained CC, CPA for WS potential for a range of frequencies. The conditions of $\mathrm{CC}$ depend on the direction of incident waves and no range exists for right incident case

for this non-Hermitian potential. More interestingly by adjusting the parameters in the potential we can have these total absorptions in any desired ranges of frequencies. Waveguide analogy of the system are constructed. It will be interesting if this result is realised in an experiment, then it will be possible to construct anti-Laser with a wide range of working frequency. We observed very interesting result in complex scattering in space fractional quantum mechanics (SFQM).The paths in the path integral formulation of standard quantum mechanics (SQM) introduced by Feynman are Brownian paths. SFQM is developed by replacing Brownian path by a more general path known as Levy flight path.The Levy flight paths are characterized by Levy index $\alpha, 0<\alpha \leq 2$. For $\alpha=2$, the Levy process are the Gaussian process. When the path integral formulation is generalized using Levy flight paths, the quantum mechanical systems are described equivalently by fractional Schrodinger equation. The fractional Schrodinger equation in one dimension is,

$i \hbar \frac{\partial \psi(x, t)}{\partial t}=H_{\alpha}(x, t) \psi(x, t)$

where $H_{\alpha}(x, t)$ is the fractional Hamiltonian operator and is expressed through Riesz fractional derivative $\left(\hbar^{2} \Delta\right)^{\alpha / 2}$ as,

$H_{\alpha}(x, t)=D_{\alpha}\left(\hbar^{2} \Delta\right)^{\alpha / 2}+V(x, t)$

We explore the features of quantum scattering in the domain of non-Hermitian space fractional quantum mechanics (NHSFQM) governed by fractional Schrodinger equation characterized by Levy index $\alpha(1<\alpha \leq 2)$. We observe that non-Hermitian SFQM systems have more flexibility for SS and CPA and display

some new features of scattering. For the delta potential $\mathrm{V}(\mathrm{x})=-\mathrm{i} \rho \delta\left(\mathrm{x}-\mathrm{x}_{0}\right), \rho>0$, the SS energy, $E_{s s}$, is blue or red shifted with decreasing $\alpha$ depending the strength of the potential. For complex rectangular barrier in non-Hermitian SQM, it is known that the reflection and transmission amplitudes are oscillatory near the spectral singular point. It is found that these oscillations eventually develop SS in non-Hermitian SFQM. The similar features is also reported for the case of CPA phenomena from complex rectangular barrier in non-Hermitian SFQM. These observations suggest a deeper relation between scattering features of non-Hermitian SQM and non-Hermitian SFQM [19].

\section{Conclusions}

The physics with non-Hermitain Hamiltonian has created a well respected position in the frontier area over the last two decades with numerous applications in different branches of physics.

In this brief note we have reviewed some of our results in two important aspects in nonHermitian physics. We have discussed the celebrated PT phase transition in higher dimensional non relativistic systems, in relativistic systems describing massless Dirac particle in the background of Dirac oscillator potential in presence of external magnetic field and in SU(N) QCD. In QCD we are able to see the deconfinement to confinement 
transition as PT phase transition, which is an extremely important realization in this field. The other aspect we include in this note is some aspects of complex scattering. So far critical coupling and CPA were achieved only for certain discrete incident energies. We for the first time provided model to construct broadband CPA, which will be very helpful to construct Anti-Laser with a wide range of operating frequencies. Blue shift and red shift are noted for SS and CPA depending on the system parameters in the complex scattering in NHSFQM.

\section{References}

[1] C. M. Bender and S. Boettcher, Phys. Rev. Lett. 80, 5243 (1998).

[2] A. Mostafazadeh, J. Math. Phys. 43, 205,(2002); J. Math.Phys.43:2814 (2002.

[3] C.M. Bender, Rep. Progr. Phys. 70, 947 (2007) and references therein.

[4] A. Mostafazadeh, Int. J. Geom. Meth. Mod. Phys. 7, 1191(2010) and references therein.

[5] Z. H. Musslimani, K. G. Makris, R. ElGanainy, and D. N. Christodoulides, Phys. Rev. Lett. 100, 030402 (2008).

[6] A. Ghatak and B. P. Mandal, J. Phys. A:

Math. Theor. 45, 355301 (2012).

[7] N Kumari, RK Yadav, A Khare, B

Bagchi, B P Mandal Annals of Physics, 373, 163 (2016).

[8] B. P. Mandal and S S. Mahajan Communication in Theoretical Physics, 64, 425 (2015).
[9] C. M. Bender, S. Boettcher, and P. N. Meisinger, J. Math. Phys. 40, 2201 (1999). [10] A. Khare and B. P. Mandal, Phys. Lett. A 272, b (2000).

[11] C. M Bender and D. J Weir,J. Phys. A: Math. Theor. 45, 425303 (2012).

[12] B. P. Mandal, B. K. Mourya, and R. K. Yadav (BHU), Phys. Lett. A 377, 1043

(2013).

[13] B. P. Mandal, B K Mourya, K Ali, A Ghatak Annals of Physics , 363, 185 (2015). [14] Haresh Rawal and B P Mandal arxiv: 1805.02510.

[15] B. P. Mandal, Mod. Phys. Lett. A 20, 655(2005).

[16] B. P. Mandal and A. Ghatak, J. Phys. A: Math. Theor. 45, 444022 (2012) .

[17] A. Ghatak, M. Hasan and B. P. Man- dal, Phys. Lett. A 379, 1326 (2015).

[18] M. Hasan, A. Ghatak and B. P. Mandal, Ann. of Phys. 344, 17 (2014).

[19] M. Hassan, B P Mandal Annals of Physics, 396, 371, (2018).

[20] N. Hatano and D. R. Nelson, Phys. Rev. Lett. 77, 570 (1996).

[21] Y. D. Chong, Li Ge, Hui Cao, and A. D. Stone Phys. Rev. Lett. 105, (2010);C. F. Gmachl, Nature 467, 37 (2010); W. Wan, Y. Chong, L. Ge, H. Noh, A. D. Stone, H. Cao, Science 331, 889 (2011); N.Liu, M. Mesch, T. Weiss, M. Hentschel, and H. Giessen, Nano Lett. 10, 2342 (2010); Y. D. Chong and A. D. Stone, Phys. Rev. Lett. 107, 163901 (2011); A. Mostafazadeh and M. Sarisaman, Proc.R. Soc. A 468, 3224 (2012). 